\title{
Identifying Methamphetamine Dependence Using Regional Homogeneity in BOLD Signals
}

\author{
Hufei Yu $\mathbb{D}^{1,2}$ Shucai Huang, ${ }^{1,3,4}$ Xiaojie Zhang, ${ }^{1,3}$ Qiuping Huang, ${ }^{1,3}$ Jun Liu, ${ }^{5}$ \\ Hongxian Chen $\mathbb{D}^{1,3}$ and Yan Tang $\mathbb{D}^{2}$ \\ ${ }^{1}$ National Clinical Research Center for Mental Disorders, and Department of Psychiatry, The Second Xiangya Hospital of Central \\ South University, Changsha, Hunan 410000, China \\ ${ }^{2}$ School of Computer Science and Engineering, Central South University, Changsha, Hunan 410000, China \\ ${ }^{3}$ Institute of Mental Health of the Second Xiangya Hospital, Central South University, Chinese National Technology Institute on \\ Mental Disorders, Hunan Key Laboratory of Psychiatry and Mental Health, Hunan Medical Center for Mental Health, Changsha, \\ Hunan 410000, China \\ ${ }^{4}$ The Fourth People's Hospital of Wuhu, Wuhu, Anhui 214000, China \\ ${ }^{5}$ Department of Medical Imaging, The Second Xiangya Hospital, Central South University, Changsha, Hunan 410000, China
}

Correspondence should be addressed to Hongxian Chen; shenhx2018@csu.edu.cn and Yan Tang; tangyan@csu.edu.cn

Received 4 September 2019; Revised 23 April 2020; Accepted 11 May 2020; Published 28 May 2020

Guest Editor: Adam Konefal

Copyright $\odot 2020$ Hufei Yu et al. This is an open access article distributed under the Creative Commons Attribution License, which permits unrestricted use, distribution, and reproduction in any medium, provided the original work is properly cited.

\begin{abstract}
Methamphetamine is a highly addictive drug of abuse, which will cause a series of abnormal consequences mentally and physically. This paper is aimed at studying whether the abnormalities of regional homogeneity (ReHo) could be effective features to distinguish individuals with methamphetamine dependence (MAD) from control subjects using machine-learning methods. We made use of resting-state fMRI to measure the regional homogeneity of 41 individuals with MAD and 42 age- and sex-matched control subjects and found that compared with control subjects, individuals with MAD have lower ReHo values in the right medial superior frontal gyrus but higher ReHo values in the right temporal inferior fusiform. In addition, AdaBoost classifier, a pretty effective ensemble learning of machine learning, was employed to classify individuals with MAD from control subjects with abnormal ReHo values. By utilizing the leave-one-out cross-validation method, we got the accuracy more than $84.3 \%$, which means we can almost distinguish individuals with MAD from the control subjects in ReHo values via machine-learning approaches. In a word, our research results suggested that the AdaBoost classifier-neuroimaging approach may be a promising way to find whether a person has been addicted to methamphetamine, and also, this paper shows that resting-state fMRI should be considered as a biomarker, a noninvasive and effective assistant tool for evaluating MAD.
\end{abstract}

\section{Introduction}

Methamphetamine is a type of synthetic stimulant that often appears white or colorless, and chronic overuse may result in dependence. There are many researchers claimed that chronic overuse of methamphetamine brings about adverse physical reactions and severe psychiatric symptoms, such as depressive disorder and dysthymic disorder [1,2], mental disease [3], and cognitive deficits [4-6], which may attribute to the reduction of dopamine transporter density that persists after use ceases $[7,8]$. Currently, the clinical diagnosis of individuals with methamphetamine dependence (MAD) is based on the abnormal presence of MA users, selfreported symptoms that are subject to their own bias. There is a lack of validated biomarkers that are highly relative to MAD. So, we proposed a machine-learning-based method that may be an effective aided diagnosis system for MAD.

Based on the blood oxygen level-dependent (BOLD) signal, resting-state functional magnetic resonance imaging (rsfMRI) is a useful method for the research of brain activity. $\mathrm{ReHo}$ is a promising method of the study of resting-state fMRI, which has been successfully used for a volume of researches of neurological diseases, such as the research of antisocial personality disorder [9], schizophrenia [10], and 
depression [11]. Hence, in this study, we utilized ReHo to find the differences between individuals with MAD and control subjects, and using the abnormal ReHo value in subjects, we initially employed AdaBoost classifier to distinguish these two groups-individuals with MAD and control subjects. AdaBoost classifier is a greatly outstanding ensemble learning method of machine learning, which even often outperforms support vector machine (SVM) [12] in some situations due to the classification results that are decided by many classifiers instead of single. We hypothesized that the abnormal areas showing in ReHo may be biomarkers for evaluating the MAD.

\section{Methods}

2.1. Data Acquisition and Preprocessing. Our nuclear magnetic resonance data were collected on the same fMRI instrument in the Department of Medical Imaging, Second Xiangya Hospital of Central South University. Scanning was performed using a 3.0T magnetic resonance imaging system from Siemens. A sponge earplug is inserted into the ear of the subject, and a soundproof ear is worn to reduce noise. The subjects were placed in a supine position, the head was placed in a fixed hood, and a foam pad was used to fix the sides of the head to reduce head movement. And during data acquisition, the subjects were asked to relax their minds, to keep their eyes closed, and to move as little as possible. Functional scans of the whole brain were acquired using a gradient echo EPI sequence; the parameters are as follows: $\mathrm{TR}=2000 \mathrm{~ms}, \mathrm{TE}=30 \mathrm{~ms}, \mathrm{FOV}=220 \mathrm{~mm}$, matrix $=64 \times 64$, flip angle $=80^{\circ}$, voxel size $=3.4 \times 3.4 \times 4 \mathrm{~mm}$, slice thickness $=4 \mathrm{~mm}$, and number of slice $=36$. We used interlayer scanning, even layers first and then odd layers, collecting 225 time points.

Data preprocessing was carried out employing Data Processing Assistant for Resting-State fMRI (DPARSF) [13] (http://www.restfmri.net) and Spm8 (https://www.fil.ion .ucl.ac.uk/spm/) on the Matlab R2017b. For each subject, because of the magnetic saturation and instability of participants, we removed the first ten scans of the fMRI time series. For the remaining images, the preprocessing procedure included slice timing, head motion correction, and spatial normalization to standard Montreal Neurological Institute template with a resampled voxel size of $3 \times 3 \times 3 \mathrm{~mm}$. Subjects with translation more than $1.5 \mathrm{~mm}$ and rotation exceeding 1.5 degrees in any direction were excluded. And then, we carried out detrending for fMRI data.

2.2. ReHo Calculation. We used DPARSF advanced edition to carry out ReHo calculation for each subject. The main idea of ReHo can be summarized as using Kendall's coefficient concordance (KCC) to measure the degree of similarity of multiple time courses [14]. The exact details of the method can be found in [15]. The KCC calculation formula at a certain point is as follows:

$$
W=\frac{\sum_{i=1}^{n}(R i)^{2}-n \times(\bar{R})^{2}}{(1 / 12) k^{2} \times\left(n^{3}-n\right)},
$$

TABLE 1: The information of subjects. MAD means methamphetamine dependence. The average duration of MA means the average months of individuals with MAD took methamphetamine.

\begin{tabular}{lcc}
\hline Types & $\begin{array}{c}\text { Individuals with } \\
\text { MAD }\end{array}$ & $\begin{array}{c}\text { Control } \\
\text { subjects }\end{array}$ \\
\hline Age (years) & $21 \sim 46$ & $20 \sim 46$ \\
Average (years) & 32.5 & 34.2 \\
Left/right-handed & $3 / 42$ & $2 / 41$ \\
Average duration of MA (months) & 60.4 & - \\
\hline
\end{tabular}

where $W$ represents the value of KCC, which is between 0 and $1, k$ means the number of voxels in a cluster, in our study, we set $k$ equals to 27 , and $n$ represents the time points of fMRI data. $R i$ means the total number of 27 voxels at the $i$ th time point, and obviously, $\bar{R}$ means the average value of $R$ $i$. A pipeline of DPARSF has the function to calculate the time series consistency of each voxel and its surrounding voxels in the brain and then obtain the KCC of the voxel. In this way, we get the KCC value of each voxel in the whole brain, and we obtained the ReHo map for every subject.

2.3. Discriminative Analysis. Between-group voxel-wise comparison of the ReHo was performed using the two-sample $t$ -test. From the result of two-sample $t$-test, we got different brain areas between individuals with MAD and control subjects. These brain regions were definite as regions of interest (ROIs). Each ROI was defined as a sphere (a radius of $6 \mathrm{~mm}$ ) whose center was at the voxel showing the highest statistical difference. Then, the mean ReHo value in each ROI for each individual participant was extracted as features to classify the MAD group and the control group. AdaBoost classification is a type of ensemble learning method of machine learning, which is proposed by Freund and Schapire [16]. The AdaBoost algorithm consists of many weak classifiers. In each iteration, a new weak classifier was added to the algorithm until the classifier reaches an expected result. During the training process, we set a value for each training sample, which represents the probability that the sample is selected by the new classifier. If the sample is accurately classified in the previous classifier, its weight will decrease, otherwise increase, so that we can pay more attention to the sample of the wrong classification. The final classification result is a linear combination of multiple classifiers, which is the fundamental reason why the AdaBoost classification algorithm is often better than others. Because of the small amount of data, we used the method of leave-one-out cross-validation to train the model, and the ultimate model accuracy is the average of multiple training.

\section{Results}

3.1. Subjects. In our study, 41 individuals with MAD come from The Forced Isolation and Detoxification Center in Pingtang, Hunan Province, China. 42 control subjects are recruited from society; we got rid of control subjects that are diagnosed with mental diseases or subjects that have 


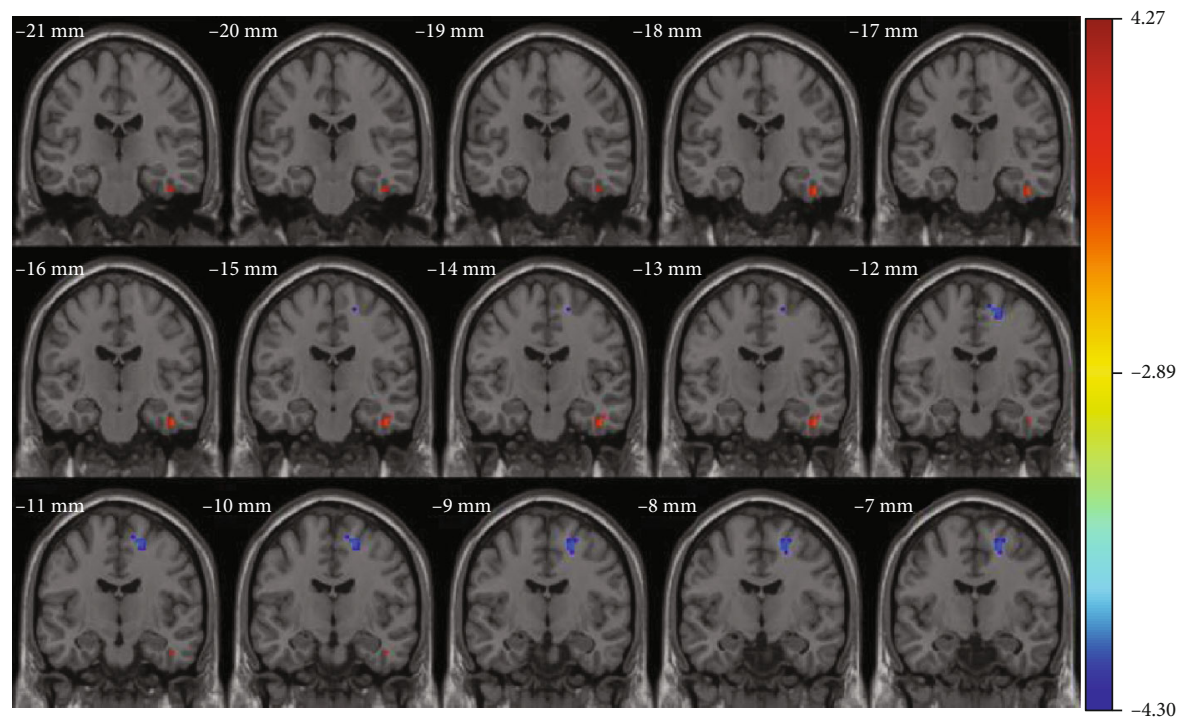

Figure 1: Significant differences in ReHo between individuals with MAD and control subjects. The parts of blue color show where ReHo value decreased in individuals with MAD compared with control subjects, and the parts of red show the increased ReHo value.

TABLE 2: Significant between-group differences in regional homogeneity.

\begin{tabular}{lcccccc}
\hline & \multicolumn{6}{c}{ Peak MNI location } \\
Regions & L/R & $x$ & $y$ & $z$ & Max $t$-value & Cluster size \\
\hline Temporal IF & $\mathrm{R}$ & 45 & -15 & -30 & 3.2546 & 14 \\
Superior FG & $\mathrm{R}$ & 18 & -12 & 57 & -3.2969 & 13 \\
\hline
\end{tabular}

Abbreviations: R: right; L: left; MNI: Montreal Neurological Institute; IF: inferior fusiform; FG: frontal gyrus.

no ability to sign their names because of poor education. There are two left-handed subjects in control subjects and three left-handed subjects in individuals with MAD. For control subjects, they have no history of addictive substance dependence except nicotine. Participants who took part in the experiment were between 20 and 46 years old with an average age of 33.4. Before the data was collected, the doctor signed a written guarantee agreement with each subject, so this study was subject to approval by the ethics committee of Central South University. The relevant information about all the participants is given in Table 1 .

3.2. ReHo Results. Figure 1 and Table 2 show meaningful differences between individuals with MAD and control subjects employing a two-sample $t$-test (after AlphaSim correction, $p=0.05$, the minimum cluster size is 13). Compared with control subjects, individuals with MAD have lower ReHo values in the right medial superior frontal gyrus, instead, and they have higher ReHo values in the right temporal inferior fusiform.

3.3. Classification Results. We used AdaBoost classifier to discriminate individuals with MAD from control subjects by features that are significant differences in ReHo between two groups. The main idea of the AdaBoost classifier can be summarized as a linear combination of multiple weaker clas- sifiers. We tested the number of weaker classifiers (n_estimators) from 2 to 20 and found that the best accuracy was $84.37 \%$ (Figure 2) when n_estimators equal to 4 . Here, accuracy means the number of correctly classified subjects divide the total number of our subjects. From the classification result, we can reach the conclusion that AdaBoost classifier can better distinguish between normal people and individuals with MAD.

\section{Discussion}

Recently, methamphetamine has been becoming one of the most highly addictive drugs in the world and continues to be foremost public health problems [17]. There is an increasing number of people that are suffering from the overuse of MA, which highly affects their physical and mental health and brings misfortune to their families. Hence, it is meaningful to identify individuals with MAD and take certain procedures to alleviate their conditions.

In this paper, our purpose was to build an assistant diagnosis system for MAD based on resting-state fMRI and machine-learning methods. In our study, we exploited the approach of ReHo to research resting-state fMRI data, by computing the discernible differences between the MAD group and the control group. In addition, an effective machine-learning method-AdaBoost algorithm-was employed to distinguish individuals with MAD and control subjects with accuracy equal to $84.3 \%$, which indicates that the AdaBoost classifier-neuroimaging approach can be a useful assistant diagnosis tool to identify individuals with MAD and help them alleviate their conditions in time. In our study, we found that individuals with MAD have lower ReHo values in their right medial superior frontal gyrus, which is consistent with the findings of Monterosso et al. and Schwartz et al. $[18,19]$. On the other hand, we found that individuals with MAD have higher ReHo values in the right temporal 


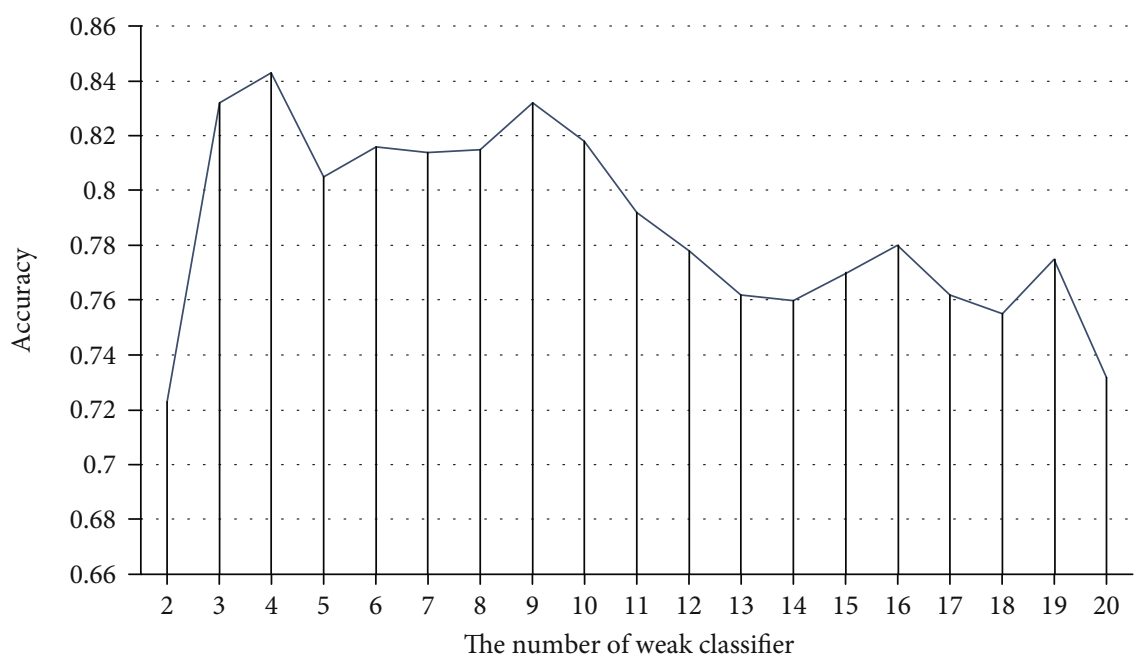

FIGURE 2: Relationship between the number of weak classifier and classify results.

inferior fusiform that is in accordance with Kim et al. [20]. Goldberg et al. found testimonies to prove that the superior frontal gyrus is relative to self-awareness [21, 22], and Fried et al. found that superior frontal gyrus is closely associated with laughter [23], which accord with clinical symptoms of individuals with MAD. And also, a series of researches proved that inferior fusiform gyrus is involved in higher processing of colors [24-26], which implies that it is often related to the visual pathway [27]; in other words, inferior fusiform gyrus is highly about face and body recognition and word recognition [28-30]; that is why most individuals with MAD often appear weird facial expressions and behaviors. Our research suggested that individuals with MAD have poor self-awareness such as compulsive behavior, anxiety, and poor ability to recognize face, body, and word [11]. In addition, we effectively distinguish individuals with MAD from control subjects using AdaBoost classifier that overrides support vector machine and KNN. Our results demonstrated a potential biomarker for evaluating MAD. There are many kinds of research showed that biomarkers might be employed to effectively diagnosis a variety of diseases in the medical domain [31]. Our results proved that ReHo could be a robust index for discriminating individuals with MAD from control subjects and machine learning could be a useful tool for the diagnosis of mental disease. Although we achieved $84.3 \%$ accuracy, we believe that we still have a big room to improve our model. And we only used regional homogeneity ( $\mathrm{ReHo}$ ) approach to estimate MAD; maybe we can transform our model in functional connectivity in the future. And also, deep learning outmatches traditional machine-learning algorithms in large sample data set in recent years, which may inspire us to get a more accurate result in the future.

\section{Data Availability}

All the data used in my paper are available; researchers who want to get data can email me at yuhufei@csu.edu.cn.

\section{Conflicts of Interest}

The authors declare that they have no conflicts of interest.

\section{Authors' Contributions}

Hufei Yu and Shucai Huang contributed equally to this work and should be considered co-first authors.

\section{Acknowledgments}

This work was supported by the National Natural Science Foundation of China (81971249), the National Basic Research Program of China (2015CB553504), and the National Research Program of China (2016YFC0800908-Z02).

\section{References}

[1] E. D. London, S. L. Simon, S. M. Berman et al., "Mood disturbances and regional cerebral metabolic abnormalities inrecently abstinent methamphetamine abusers," Archives of General Psychiatry, vol. 61, no. 1, pp. 73-84, 2004.

[2] T. F. Newton, A. D. Kalechstein, S. Duran, N. Vansluis, and W. Ling, "Methamphetamine abstinence syndrome: preliminary findings," The American Journal on Addictions, vol. 13, no. 3, pp. 248-255, 2004.

[3] K. M. Grant, T. D. LeVan, S. M. Wells et al., "Methamphetamine-associated psychosis," Journal of Neuroimmune Pharmacology, vol. 7, no. 1, pp. 113-139, 2012.

[4] A. C. Dean, S. M. Groman, A. M. Morales, and E. D. London, "An evaluation of the evidence that methamphetamine abuse causes cognitive decline in humans," Neuropsychopharmacology, vol. 38, no. 2, pp. 259-274, 2013.

[5] A. D. Kalechstein, T. F. Newton, and M. Green, "Methamphetamine dependence is associated with neurocognitive impairment in the initial phases of abstinence," The Journal of Neuropsychiatry and Clinical Neurosciences, vol. 15, no. 2, pp. 215-220, 2003.

[6] C. C. Cruickshank and K. R. Dyer, "A review of the clinical pharmacology of methamphetamine," Addiction, vol. 104, no. 7, pp. 1085-1099, 2009. 
[7] Y. Sekine, M. Iyo, Y. Ouchi et al., "Methamphetaminerelated psychiatric symptoms and reduced brain dopamine transporters studied with PET," American Journal of Psychiatry, vol. 158, no. 8, pp. 1206-1214, 2001.

[8] N. D. Volkow, L. Chang, G.-J. Wang et al., "Association of dopamine transporter reduction with psychomotor impairment in methamphetamine abusers," American Journal of Psychiatry, vol. 158, no. 3, pp. 377-382, 2001.

[9] Y. Tang, W. Liu, J. Chen, J. Liao, D. Hu, and W. Wang, "Altered spontaneous activity in antisocial personality disorder revealed by regional homogeneity," Neuroreport, vol. 24, no. 11, pp. 590-595, 2013.

[10] H. Liu, Z. Liu, M. Liang et al., "Decreased regional homogeneity in schizophrenia: a resting state functional magnetic resonance imaging study," NeuroReport, vol. 17, no. 1, pp. 19-22, 2006.

[11] Z. Ma, R. Li, J. Yu, Y. He, and J. Li, "Alterations in regional homogeneity of spontaneous brain activity in late-life subthreshold depression," PloS one, Y.-F. Zang, Ed., vol. 8, no. 1, 2013.

[12] C. Cortes and V. Vapnik, "Support-vector networks," Machine Learning, vol. 20, no. 3, pp. 273-297, 1995.

[13] Y. Chao-Gan and Z. Yu-Feng, "DPARSF: a MATLAB toolbox for "pipeline" data analysis of resting-state fMRI," Frontiers in systems neuroscience, vol. 4, 2010.

[14] J. L. Müller, S. Gänßbauer, M. Sommer et al., "Gray matter changes in right superior temporal gyrus in criminal psychopaths. Evidence from voxel-based morphometry," Psychiatry Research: Neuroimaging, vol. 163, no. 3, pp. 213-222, 2008.

[15] L. Ni, R. Qi, L. J. Zhang et al., “Altered regional homogeneity in the development of minimal hepatic encephalopathy: a resting-state functional MRI study," PloS one, vol. 7, no. 7, p. e42016, 2012.

[16] Y. Freund and R. E. Schapire, "A desicion-theoretic generalization of on-line learning and an application to boosting," in European Conference on Computational Learning Theory, pp. 23-37, Barcelona, Spain, 1995.

[17] E. D. London, M. Kohno, A. M. Morales, and M. E. Ballard, "Chronic methamphetamine abuse and corticostriatal deficits revealed by neuroimaging," Brain Research, vol. 1628, pp. 174-185, 2015.

[18] J. R. Monterosso, G. Ainslie, J. Xu, X. Cordova, C. P. Domier, and E. D. London, "Frontoparietal cortical activity of methamphetamine-dependent and comparison subjects performing a delay discounting task," Human Brain Mapping, vol. 28 , no. 5, pp. 383-393, 2007.

[19] D. L. Schwartz, A. D. Mitchell, D. L. Lahna, et al., "Global and local morphometric differences in recently abstinent methamphetamine-dependent individuals," NeuroImage, vol. 50, no. 4, pp. 1392-1401, 2010.

[20] Y. T. Kim, H. J. Song, J. H. Seo et al., “The differences in neural network activity between methamphetamine abusers and healthy subjects performing an emotion-matching task: functional MRI study," NMR in Biomedicine, vol. 24, no. 10, pp. 1392-1400, 2011.

[21] I. I. Goldberg, M. Harel, and R. Malach, "When the brain loses its self: prefrontal inactivation during sensorimotor processing," Neuron, vol. 50, no. 2, pp. 329-339, 2006.
[22] S. M. Platek, J. P. Keenan, G. G. Gallup Jr., and F. B. Mohamed, "Where am I? The neurological correlates of self and other," Cognitive Brain Research, vol. 19, no. 2, pp. 114-122, 2004.

[23] I. Fried, C. L. Wilson, K. A. MacDonald, and E. J. Behnke, "Electric current stimulates laughter," Nature, vol. 391, no. 6668, pp. 650-650, 1998.

[24] V. S. Ramachandran, The Tell-Tale Brain: Unlocking the Mystery of Human Nature: Random House, 2012.

[25] M. Corbetta, F. Miezin, S. Dobmeyer, G. Shulman, and S. Petersen, "Attentional modulation of neural processing of shape, color, and velocity in humans," Science, vol. 248, no. 4962, pp. 1556-1559, 1990.

[26] W. K. Simmons, V. Ramjee, M. S. Beauchamp, K. McRae, A. Martin, and L. W. Barsalou, "A common neural substrate for perceiving and knowing about color," Neuropsychologia, vol. 45, no. 12, pp. 2802-2810, 2007.

[27] E. M. Hubbard and V. S. Ramachandran, "Neurocognitive mechanisms of synesthesia," Neuron, vol. 48, no. 3, pp. 509520, 2005.

[28] N. George, R. J. Dolan, G. R. Fink, G. C. Baylis, C. Russell, and J. Driver, "Contrast polarity and face recognition in the human fusiform gyrus," Nature Neuroscience, vol. 2, no. 6, pp. 574$580,1999$.

[29] S. Bölte, D. Hubl, S. Feineis-Matthews, D. Prvulovic, T. Dierks, and F. Poustka, "Facial affect recognition training in autism: can we animate the fusiform gyrus?," Behavioral Neuroscience, vol. 120, no. 1, pp. 211-216, 2006.

[30] B. D. McCandliss, L. Cohen, and S. Dehaene, "The visual word form area: expertise for reading in the fusiform gyrus," Trends in Cognitive Sciences, vol. 7, no. 7, pp. 293-299, 2003.

[31] X. Chen, Y. Ba, L. Ma et al., "Characterization of microRNAs in serum: a novel class of biomarkers for diagnosis of cancer and other diseases," Cell Research, vol. 18, no. 10, pp. 9971006, 2008. 